\title{
Combined Automatic Lane-Keeping and Driver's Steering Through a 2-DOF Control Strategy
}

\author{
Vito Cerone, Member, IEEE, Mario Milanese, Senior Member, IEEE, and Diego Regruto, Member, IEEE
}

\begin{abstract}
In this paper, we address the problem of combining automatic lane-keeping and driver's steering for either obstacle avoidance or lane-change maneuvers for passing purposes or any other desired maneuvers, through a closed-loop control strategy. The automatic lane-keeping control loop is never opened, and no on/off switching strategy is used. During the driver's maneuver, the vehicle lateral dynamics are controlled by the driver himself through the vehicle steering system. When there is no driver's steering action, the vehicle center of gravity tracks the center of the traveling lane thanks to the automatic lane-keeping system. At the beginning (end) of the maneuver, the lane-keeping task is released (resumed) safely and smoothly. The performance of the proposed closed-loop structure is shown both by means of simulations and through experimental results obtained along Italian highways.
\end{abstract}

Index Terms-automatic lane-keeping, driver's steering, lanechange, two-degrees-of-freedom (2-DOF) control, vehicle lateral control.

\section{INTRODUCTION}

I NTELLIGENT vehicle systems (IVSs) have recently become an attractive area of research throughout the world. The aim of the research effort is mainly that of enhancing driving safety and reducing the driver's workload. In [1], the positive effect of driver assistance systems on drivers' physical and mental workload reduction is shown through the measure of some vital reactions such as the electromyographic and the electrodermal activities. In particular, automated highway systems (AHS), which have been extensively studied at Ohio State University since 1964 [2], are receiving renewed attention due to fast developments in hardware/software technology that stimulate the design of more effective control systems. Since the mid-1980s, a larger effort is being conducted mainly in the California PATH program. Early attempts of the project were devoted to assess previous knowledge in the field of automatic vehicle control providing the analytical basis for new developments [3]. In the last several years, large efforts have been directed to the solution of the highway automatic steering control problem for different types of vehicles and using different control strategies and techniques. Most of the

Manuscript received March 09, 2007; revised October 31, 2007. Manuscript received in final form April 10, 2008. First published June 13, 2008; current version published December 24, 2008. Recommended by Associate Editor A. Stefanopoulou. This work was supported in part by the Italian Ministero dell'Istruzione, dell'Università e della Ricerca (MIUR) under the plan "Control of advanced systems of transmission, suspension, steering and braking for the management of the vehicle dynamics" and by Centro Ricerche Fiat (CRF) under Contract "Analisi e sintesi di nuove architetture di controllo laterale per il mantenimento della corsia di marcia di un autoveicolo."

The authors are with the Dipartimento di Automatica e Informatica, Politecnico di Torino, 10129 Torino, Italy (e-mail: vito.cerone@ polito.it; mario. milanese@polito.it; diego.regruto@polito.it).

Digital Object Identifier 10.1109/TCST.2008.924558 contributions rely on buried magnet or electrified wires placed along the path for the detection of the vehicle lateral position, the so-called look down sensing scheme. The problem, in the case of passenger cars, was analyzed in this framework by Patwardhan et al. in [4], where they show the fundamental control difficulties of this approach. An interesting alternative approach, that avoids the modification of infrastructures, involves the use of vision sensors placed on the vehicle, the so-called look-ahead sensing scheme. A comparative study of vision-based control strategies was presented by Košeckà et $a l$. in [5]. A great deal of remarkable works about application of advanced linear and nonlinear control techniques to the automatic steering control problem were conducted, still in the PATH program, by Tomizuka and coworkers in the case of four wheeled vehicles in [6], heavy vehicles in [7] and [8], and tractor-semitrailer combinations in [9], while in [10] and [11] the problem of designing a fault-tolerant lane-keeping controller for automated vehicles has been considered. In recent years, the problem of steering control has attracted wide interest also outside the PATH program. Relevant contributions in the field of robust steering and vehicle lateral dynamics control were also provided by Ackermann and coworkers (see, e.g., [12] and [13]). Preliminary experimental results on robust lateral control conducted by Byrne et al. were reported in [14], highlighting several implementation difficulties. Adaptive steering control for lane-keeping of a tractor-semitrailer has been proposed by Wang et al. in [15], while a model-predictive control strategy for lateral control of autonomous vehicles has been considered in [16]. Other contributions based on the look-ahead sensing scheme were provided by Hatipoğlu et al. in [17], where they used a digital videocamera together with a radar system, by Broggi et al. [18], who used a stereo vision system composed of two videocameras, and by Cerone et al. [19]-[21], where properties of single-input two-output (SITO) systems are exploited. Significant Japanese contributions to the development of vision-based intelligent vehicles, given since the mid 1970s to the early 1990s, are surveyed by Tsugawa [22].

Some of the advanced maneuvers pertaining to vehicle lateral control are lane changing for vehicle-passing purposes and obstacle avoidance. The problem of automated lane-change maneuvers is widely addressed in the literature. In [17], [23], and [24], for example, the authors consider open-loop and closedloop lane-change and design time optimal steering controllers with nonlinear constraints. First, they generate a special openloop lane-change steering signal which minimizes the period of lane-change subject to constraints on the lateral acceleration and jerk. Then, they discuss how to implement those steering commands in the closed-loop system using a lane-following controller previously published. We point out that autonomous lane 
change deals with the generation of the appropriate steering signal to have the vehicle accomplish the task without driver assistance.

In this paper, we address the problem of combining automatic lane-keeping and driver's steering for either obstacle avoidance or lane-change maneuvers for passing purposes or any other desired maneuvers through a closed-loop control strategy. The proposed control strategy is designed assuming that the vehicle is traveling along highway paths. First, in Section II, we present the physical plant and derive a suitable simplified model focusing on the accordance between simplification hypotheses and experimental context. Then, in Section III, we formulate the control problem. Further, in Section IV, we propose a two-degree-of-freedom (2-DOF) closed-loop control strategy that gives a solution to the formulated problem. The automatic lane-keeping control loop is never opened, and no on/off switching strategy is used. During the driver's maneuver, the vehicle lateral dynamics are controlled by the driver himself through the vehicle steering system. When there is no driver steering action, the vehicle's center of gravity tracks the center of the traveling lane thanks to the automatic lane-keeping system. At the beginning (end) of the maneuver, the lane-keeping task is released (resumed) safely and smoothly. Both simulation and experimental results along Italian highways, obtained with the proposed 2-DOF structure on a FIAT Brava 1600 ELX provided by Centro Ricerche Fiat, are presented and discussed in Sections V and VI, respectively.

\section{Plant DESCRIPTION AND MODELING}

The plant to be controlled, provided by Centro Ricerche Fiat, consists of a Fiat Brava 1600 ELX equipped with a vision system and a steering actuator. The vision system comprises a single CCD videocamera and related image-processing algorithms. The steering actuator system is a locally controlled dc brushless electric motor. Both control and vision algorithms are processed by an INTEL 486 microprocessor-based PC for industrial applications. The sampling time of the whole system is $T_{s}=40 \mathrm{~ms}$. The described hardware is not supposed to be modifiable by the control designer. The mathematical modeling of such a plant was discussed in detail in [19], in which a simplified model that is suitable for the description of the vehicle behavior in highway experimental conditions was presented. The equations of such a model are recalled here for self-consistency of the paper. The interaction between the vehicle lateral dynamics and the vision system can be modeled by the following state space equations parameterized by the longitudinal velocity $v_{x}$ :

$$
\begin{aligned}
{\left[\begin{array}{c}
\dot{v}_{y} \\
\ddot{\psi} \\
\dot{q} \\
\dot{m}
\end{array}\right]=} & {\left[\begin{array}{cccc}
\frac{-a_{1}}{m_{v} v_{x}} & \frac{-m_{v} v_{x}^{2}+a_{2}}{m_{v} v_{x}} & 0 & 0 \\
\frac{a_{3}}{I_{\psi} v_{x}} & \frac{-a_{4}}{I_{\psi} v_{x}} & 0 & 0 \\
-1 & 0 & 0 & v_{x} \\
0 & -1 & 0 & 0
\end{array}\right]\left[\begin{array}{c}
v_{y} \\
\dot{\psi} \\
q \\
m
\end{array}\right] } \\
& +\left[\begin{array}{c}
b_{1} \\
b_{2} \\
0 \\
0
\end{array}\right] \delta_{v}+\left[\begin{array}{c}
0 \\
0 \\
-L v_{x} \\
v_{x}
\end{array}\right] K_{L}
\end{aligned}
$$

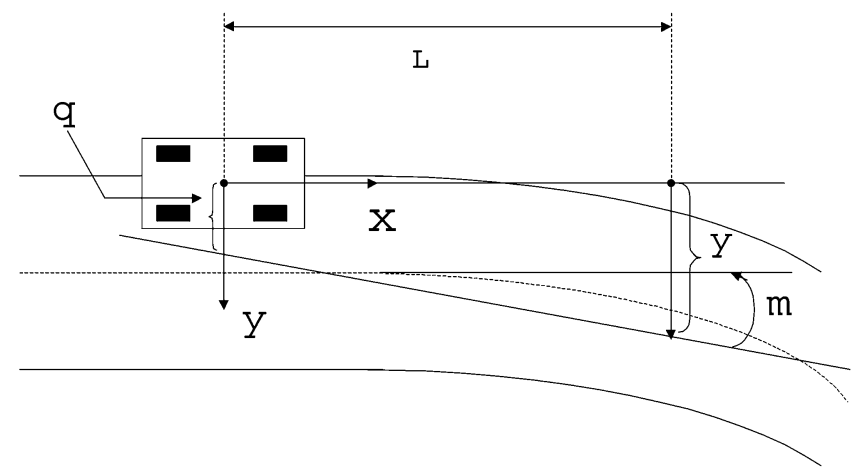

Fig. 1. Feedback signal $y$.

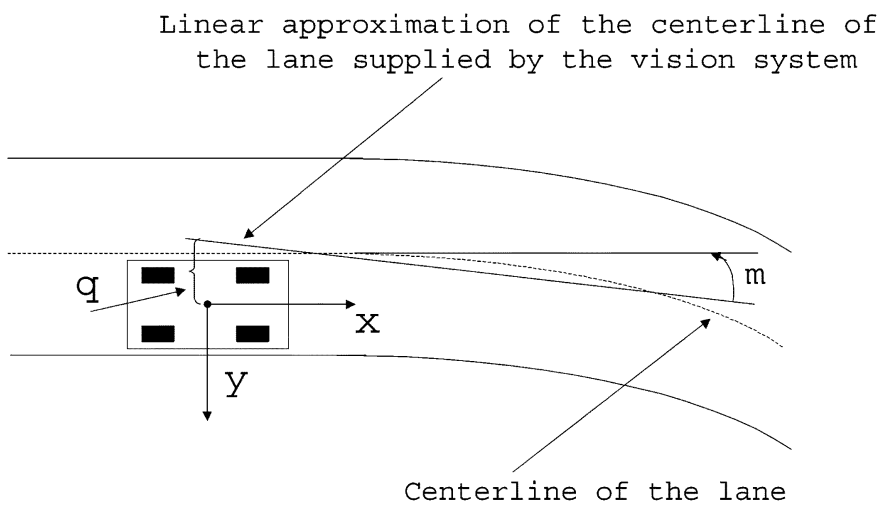

Fig. 2. Centerline linear approximation supplied by the vision system.

where $L$ is the so-called look-ahead distance, taken along the longitudinal axis, from the vehicle center of gravity to a suitable point in the road between 3-20 m ahead of the vehicle (see Fig. 1), $K_{L}(t)$ is the road curvature, i.e., the inverse of the instantaneous curve radius at the look-ahead point, $\delta_{v}$ is the steering-wheel angle, and $m$ and $q$ are the measurements supplied by the vision system about the vehicle location on the lane, as shown in Fig. 2. The meanings of the symbols involved are

$m_{v}$ vehicle mass;

$v_{x}$ longitudinal component of center of gravity (CG) velocity;

$v_{y}$ transverse component of $\mathrm{CG}$ velocity;

$\psi \quad$ vehicle yaw angle;

$I_{\psi} \quad$ inertial vehicle moment around center of gravity referred to the vertical axis;

$\delta_{v} \quad$ steering-wheel angle;

$k$ front wheels angle/steering-wheel angle ratio, expressed in $\mathrm{rad} /$ degrees;

$\delta_{f}=\delta_{v} k$ : front wheels angle;

$c_{r} \quad$ cornering stiffness of front tires;

$c_{f} \quad$ cornering stiffness of rear tires;

$l_{r} \quad$ distance between the rear axle and the center of gravity;

$l_{f} \quad$ distance between the front axle and the center of gravity;

$l=l_{r}+l_{f}:$ wheelbase;

$a_{1}=c_{f}+c_{r} ;$

$a_{2}=c_{r} l_{r}-c_{f} l_{f}$; 


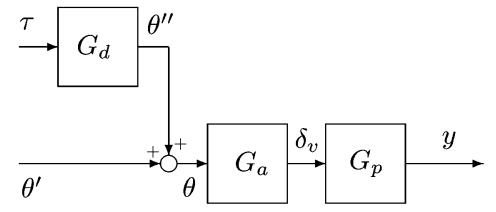

Fig. 3. Plant block diagram.

$$
\begin{aligned}
a_{3} & =-l_{f} c_{f}+l_{r} c_{r} ; \\
a_{4} & =l_{f}^{2} c_{f}+l_{r}^{2} c_{r} ; \\
b_{1} & =\frac{c_{f}}{m} k ; \\
b_{2} & =\frac{l_{f} c_{f}}{I_{\psi}} k .
\end{aligned}
$$

The steering actuator, given by Centro Ricerche Fiat, is described by the following transfer function:

$$
G_{a}=\frac{\delta_{v}(s)}{\theta(s)}=\frac{1580}{s^{2}+75.5 s+1580}
$$

where $\theta$ is the reference steering angle due to the sum of $\theta^{\prime}$ (provided by the lateral dynamics controller) and $\theta^{\prime \prime}$ (i.e., the effect of the torque $\tau$ applied by the driver on the steer), as can be seen in Fig. 3. The transfer function between the torque $\tau$ applied by the driver on the steer and the reference steering angle is denoted by $G_{d}$ (see Fig. 3). The bandwidth of $G_{d}(j \omega)$ is supposed to be wider than the bandwidth of the control system to be designed; from available data, it can be assumed that, in the working frequency range, a static gain $\bar{G}_{d}=1 / 3$ can be used to describe the behavior of $G_{d}$. The transfer function of the nominal plant to be controlled is $G_{p} \triangleq y(s) / \delta_{v}(s)$, where $y(t)=q(t)+m(t) L$ is the position error of the vehicle with respect to the lane centerline measured at the look-ahead distance $L$ (see [19] for a detailed discussion about the advantages of using such a quantity as feedback signal in lane-keeping control systems). The value of the look-ahead distance $L$ has to be carefully chosen taking into account the specific behavior of the vehicle to be controlled (e.g., understeering behavior, oversteering behavior, neutral steering behavior, etc.). In the experimental tests performed with the vehicle under consideration, we noted that the increase of $L$ improves comfort performances though it tends to cause large lateral position error at the center of gravity (CG) when a curve is approached. The experimental results presented in this paper were obtained with $L=11.5 \mathrm{~m}$. Although such a value, obtained through trial and error, provides good results for the specific vehicle considered in this study, we remark that the problem of choosing the value of $L$ may require deeper analysis in the general case. Most of the early contributions in the field of steering control were based on the use of reduced-order nominal models like the one presented above, sometimes considering the effect of the longitudinal velocity variations (see, for example, [25]). In recent years, works have been presented where the attention is focused on the model uncertainty (see, e.g., [12], [14], [13], and [7]). Looking at the proposed model, it can be noted that some unmodeled linear

\begin{tabular}{|c|c|c|c|}
\hline & Parameter & $\gamma_{\text {nom }}$ & PUI \\
\hline$\gamma_{1}$ & $m_{v}(\mathrm{~kg})$ & 1226 & {$[1226,1626]$} \\
\hline$\gamma_{2}$ & $I_{\psi}\left(\mathrm{kgm}^{2}\right)$ & 1900 & {$[1900,2520]$} \\
\hline$\gamma_{3}$ & $c_{r}(\mathrm{~N} / \mathrm{rad})$ & 96000 & {$[81600,110400]$} \\
\hline$\gamma_{4}$ & $c_{f}(\mathrm{~N} / \mathrm{rad})$ & 60000 & {$[51000,69000]$} \\
\hline & $l_{r}(\mathrm{~m})$ & 1,506 & - \\
\hline & $l_{f}(\mathrm{~m})$ & 1,034 & - \\
\hline$\gamma_{5}$ & $v_{x}(\mathrm{~km} / \mathrm{h})$ & 95 & {$[60,120]$} \\
\hline & $k$ (rad/degrees) & $\pi /(180 \cdot 18)$ & - \\
\hline
\end{tabular}
and nonlinear dynamics are present in the actual plant like, for example, roll, yaw, and heave effects neglected by the single
TABLE I

VALUES OF MODEl PARAMETERS

track model, steering gear backlash, or actuator voltage command saturation. Moreover, the system has a time-varying nature due to parametric dependence on longitudinal velocity $v_{x}$ and, finally, some parameters of the simplified model cannot be exactly known. Thus, all of these sources of uncertainty should be taken into account in the controller design. However, it can be noted that unmodeled dynamics are little excited along highway paths, which are characterized by bends with large radii requiring slow steering actions, while highway longitudinal velocity variations are typically slow. Thus, all considered, the following parametric uncertainties have been considered. The vehicle mass $m_{v}$ can take values from $1226 \mathrm{~kg}$ (nominal value) to $1626 \mathrm{~kg}$, corresponding to the mass of a vehicle with five passengers, each one weighting $80 \mathrm{~kg}$, on board. The inertial moment $I_{\psi}$ can vary coherently from $1900 \mathrm{~kg} \cdot \mathrm{m}^{2}$ to $2520 \mathrm{~kg} \cdot \mathrm{m}^{2}$. The cornering stiffness coefficients $c_{f}$ and $c_{r}$ range respectively between [51000, 69000] N/rad and [81600, 110400] N/rad. Finally, the velocity is handled as an uncertain parameter whose value belongs to $[60,120] \mathrm{km} / \mathrm{h}$. The nominal values of the parameters $\gamma_{\text {nom }}=\left[m_{v_{0}} I_{\psi_{0}} c_{r_{0}} c_{f_{0}} v_{x_{0}}\right]$ and the corresponding parameter uncertainty intervals $\mathrm{PUI}_{i} \triangleq\left[\gamma_{i}^{\min }, \gamma_{i}^{\max }\right]$ are summarized in Table I. The uncertain system generated by the given parameter uncertainty intervals can be represented by the following model set:

$$
\mathcal{M}_{P}=\left\{G_{p}(s, \gamma) \in \mathcal{C}: \gamma \in \Gamma\right\}
$$

where $\gamma=\left[\begin{array}{lllll}m_{v} & I_{\psi} & c_{r} & c_{f} & v_{x}\end{array}\right]$ is the vector of the uncertain parameters and $\Gamma=\left\{\gamma \in \Re^{5}: \gamma_{i} \in \mathrm{PUI}_{i}, i=1,2,3,4,5\right]$.

\section{CONTROL Problem Formulation}

The problem we are dealing with in this paper is the design of a closed-loop control system able to keep the vehicle inside the lane along typical highway paths and to permit any intervention on the side of the driver in order to override the automatic lane-keeping system and obtain complete control of the vehicle lateral dynamics. In other words, we aim at a combined automatic lane-keeping and driver's steering through a closed-loop control strategy. We assume that the automatic lane-keeping system is available (see, e.g., [19]-[21]). In the paper, the terms driver's steering or driver's maneuver mean any intervention of the driver on the vehicle steering system in order to obtain a desired behavior of the vehicle (for example, lane change for passing purposes or obstacle avoidance) and, in general, when it is desired to override the automatic lane-keeping and obtain complete control of the vehicle lateral dynamics.

In the paper, it is assumed that all of the signals in the control loop are seamless. As a matter of fact, as is well known, 
such an assumption is not always satisfied in practice since the nonideal behavior of the vision sensor can affect the signal integrity in some particular situations (e.g., high exposure in the CCD camera or high contrast items on the road). In addition, the presence of vibrations and noise on the sensing system can deteriorate the quality of the measured signal. Although such complications should be carefully taken into account in order to ensure that the system will work without fail, they will not be explicitly considered in this work whose contribution is focused on the development of a new control strategy to combine automatic lane keeping and driver's steering.

Control problem description-The following specifications (S1)-(S5) concur in the definition of the control problem under consideration, i.e., the design of a closed-loop control strategy for combined automatic lane keeping and driver's steering.

(S1) Before the driver's steering action, the vehicle CG tracks the center of the traveling lane thanks to the lanekeeping control system.

(S2) At the beginning of the driver's maneuver, the lanekeeping task must be released safely and smoothly.

(S3) During the driver's maneuver, the vehicle lateral dynamics are controlled by the driver himself through the vehicle steering system.

(S4) At the end of the driver's maneuver, the CG tracks the center of the lane in which the vehicle is traveling and the lane-keeping task must be resumed safely and smoothly.

(S5) A closed-loop control strategy is sought which combines the automatic lane-keeping and the driver's maneuvers, which means that the automatic lane-keeping control loop is never opened, and no on/off switching strategy is used.

Specification (S5) is introduced to guarantee the driver's safety. As a matter of fact, thanks to such a specification, the lane-keeping control loop is never physically disconnected from the plant. Therefore, if the driver accidentally loses control of the steering wheel while performing a maneuver, the vehicle will be kept inside the lane by the lane-keeping control loop.

\section{Combined Automatic LANE-KeEPING AND DRIVER's STEERING}

\section{A. 2-DOF Structure}

It is desired that the automatic lane-keeping control loop be never switched off, i.e., the loop control be always active. Furthermore, it is also specified that the transition between the automatic lane-keeping mode and the driver's steering mode be actuated without on/off switching strategy. In order to meet the above requirements, we propose the 2-DOF structure of Fig. 4. The feedback controller $C_{1}$ is designed in order to satisfy automatic lane keeping specifications (see, e.g., [19]-[21]), while $C_{2}$ is designed with the aim of combining the above described two modes in a smoothly way.

\section{B. Design of Controller $C_{2}$}

The guidelines for the design of controller $C_{2}$ are derived from specifications (S1)-(S4), which describe the control problem under consideration. More precisely, $C_{2}$ must be designed in such a way that the following conditions hold.

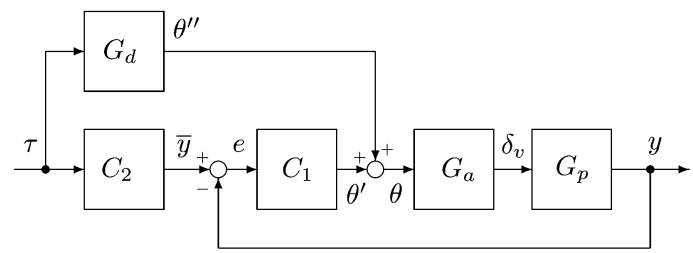

Fig. 4. Combined automatic lane-keeping and driver's steering through a 2-DOF structure.

Condition (1): the vehicle lateral dynamics are controlled by the automatic lane-keeping system when the torque $\tau$ applied by the driver on the steer is negligible $(\approx 0)$ and Condition (2): when the driver's torque $\tau$ on the steer is different from zero, the vehicle behavior perceived by the driver is as close as possible to the one of the vehicle without automatic lane-keeping.

It is easily seen that the 2-DOF structure shown in Fig. 4 takes care of condition (1). As a matter of fact, when $\tau=0$, the proposed structure simplifies to a 1-DOF structure with regulation to a zero reference signal, i.e., the automatic lane-keeping control system. In order to take care of condition (2), the following result is given.

Result 1: Condition (2) holds if and only if (iff)

$$
C_{2}=G_{d} G_{a} G_{p}
$$

Proof: $\Rightarrow$ Condition (2) implies that the transfer function $(e / \tau)$ be zero, i.e.,

$$
\frac{e}{\tau}=\frac{C_{2}}{1+C_{1} G_{a} G_{p}}-\frac{G_{d} G_{a} G_{p}}{1+C_{1} G_{a} G_{p}}=0
$$

which in turn implies (4).

$\Leftarrow$ The converse is also true. If $C_{2}=G_{d} G_{a} G_{p}$, then $(e / \tau)=0$, and the automatic lane-keeping control loop is open.

\section{Implementation Issues: Stability of $C_{2}$}

As can be seen from Fig. 4, $C_{2}$ is a cascade filter, thus it must enjoy the stability property. While $G_{d}$ is a constant scalar transfer function (t.f.) and $G_{a}$ is the stable actuator t.f., unfortunately $G_{p}$ shows two integrators responsible for instability. Thus, $C_{2}$ cannot be implemented in the form of (4). We implemented the following stable approximation of $C_{2}$ :

$$
\tilde{C}_{2}=G_{d} G_{a} \frac{s^{2}}{(s-\alpha)^{2}} G_{p}
$$

As can be seen, the double integrator contained in $G_{p}$ is replaced by a couple of real poles $s=\alpha$, and $\alpha$ is chosen in order to get an acceptable compromise between low-frequency behavior approximation and settling time of the control system step response. Results presented in the paper are obtained with $\alpha=-0.2513$.

Remark: We point out that, if controller $C_{2}$ is given by (4), at least in principle a null signal error $e$ is expected when the driver's torque $\tau$ on the steer is different from zero. However, there are a couple of reasons which lead to a nonperfect zero $e$. First, the components of $C_{2}$, i.e., $G_{d}, G_{a}$, and $G_{p}$, are not exactly known and can only be suitably approximated. Second, as 
discussed above, only a stable approximation of $G_{p}$ can be implemented. In practice, however, the presence of an error signal $e$ slightly different from zero when $\tau \neq 0$ does not compromise the desired performance of the proposed control structure. The only resulting drawback (which indeed might not be considered as such) is the presence of an "opposing" torque "felt" on the steer by the driver, which actually can be considered as a simulation of the load due to the steering system.

\section{Implementation Issues: Lane Change Maneuver}

We recall that $q$ is the measurement supplied by the vision system about the vehicle location on the lane, as shown in Fig. 2. Thus, during the lane-change maneuver, the vision system is subject to a change in the measurement reference system: before the maneuver $q$ is measured with respect to the current lane, at the end of the maneuver $q$ is measured with respect to the new lane. The transition from one lane to the next can be handled by properly resetting the initial condition of controller $C_{2}$ with respect to the new measurement reference system. This can be simply accomplished if one notes that, when the vehicle crosses the line between two lanes, there is no loss of continuity in the time evolution of the state variables $m, \dot{\psi}$, and $v_{y}$, while there is a discontinuity in the state variable $q$, as large as the width of the lane, due to the change of the measurement reference system. Thus, in order to reset the controller, a realization is sought in the state variables form, four of which are just the physical state variables $\left(m, \dot{\psi}, v_{y}\right.$, and $\left.q\right)$ of the plant, whose model $G_{p}$ is part of $C_{2}$. It can be easily shown that the controller reset can be achieved by changing the sign of the state variable of $C_{2}$ corresponding to the physical state variable $q$.

\section{E. Robustness Issues: Stability}

In order to analyze the robustness of the proposed control system, a suitable but conservative description of the uncertainty generated by the considered parameters perturbation is provided by the following model set expressed in the so-called input multiplicative form:

$$
\begin{aligned}
\mathcal{M}_{G_{p}}=\left\{G_{p}(s)=\left(1+\Delta_{m}(s)\right) G_{p_{n}}(s)\right. & : \\
\left|\Delta_{m}(j \omega)\right| & \left.\leq W_{2}(j \omega), \forall \omega\right\}
\end{aligned}
$$

where $G_{p_{n}}$ is the nominal plant, $\Delta_{m}(s)$ is a complex function which represents the unknown modeling error, and $W_{2}(s)$ is a known function bounding the modeling error. A description of $\Delta_{m}(j \omega)$ obtained gridding the hypercube $\Gamma$ is shown in Fig. 5 together with the upper bound $W_{2}(s)=0.6$. As far as robust stability is concerned, the application of the Small Gain Theorem to the block diagram of Fig. 4 leads to the following condition.

Result 2: The control system in Fig. 4 with $G_{p}(s) \in \mathcal{M}_{G_{p}}$ is robustly stable iff

$$
\left\|\frac{G_{p_{n}} G_{a} C_{1} W_{2}}{1+G_{p_{n}} G_{a} C_{1} G_{m}}\right\|_{\infty}<1
$$

where $\|\cdot\|_{\infty}$ is the $H_{\infty}$ norm of a system.

Result 2 shows that robust stability of the proposed control scheme is not affected by the design of the filter $C_{2}$. The lanekeeping controller $C_{1}(s)$ designed in [21] satisfies (8).

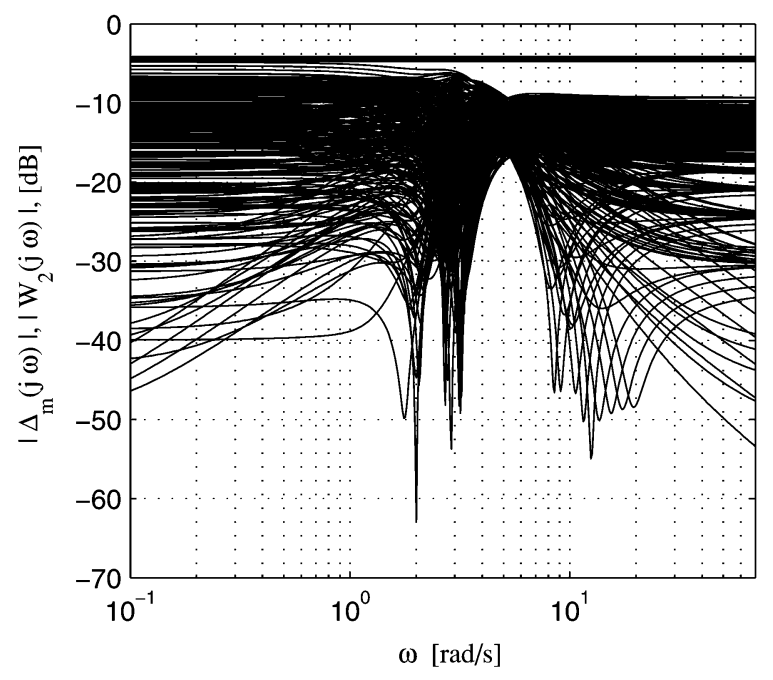

Fig. 5. Uncertainty $\Delta_{m}$ and weighting function $W_{2}$ (solid line).

\section{F. Robustness Issues: Performance}

As far as robust performance is concerned, we are interested in evaluating the effect of the modeling error on the main task of the proposed approach, expressed by (2), which is equivalent to the condition $e(s) / \tau(s)=0$. The plant is assumed to be described by the following uncertain model $G_{p}(s)=G_{p_{n}}(s)+\Delta_{a}(s)$ where $\Delta_{a}(s)=G_{p_{n}}(s) \Delta_{m}(s)$ is the additive unstructured modeling error. According to the discussion of Section IV-C, the stable approximation $\tilde{C}_{2}=G_{d} G_{a}\left(s^{2} /(s-\alpha)^{2}\right) G_{p_{n}}$ is considered for the feedforward filter $C_{2}(s)$. First of all, in order to evaluate the performance of the controlled system in the uncertainty-free case, let us compute the transfer function $e(s) / \tau(s)$ when $\Delta_{a}(s)=0$. Such a transfer function, called $H_{1}$, is given by

$$
\begin{aligned}
H_{1}(s) & =\frac{\tilde{C}_{2}}{1+C_{1} G_{a} G_{p_{n}}}-\frac{G_{d} G_{a} G_{p_{n}}}{1+C_{1} G_{a} G_{p_{n}}} \\
& =S G_{d} G_{a} G_{p_{n}}\left(\frac{s^{2}}{(s-\alpha)^{2}}-1\right)
\end{aligned}
$$

where $S=\left(1+G_{p} G_{a} C_{1}\right)^{-1}$ is the sensitivity function of the lane-keeping control loop. As expected, $H_{1} \neq 0$ since the ideal $C_{2}$ of (4) has been approximated by the filter $\tilde{C}_{2}$. However, $H_{1}$ tends to 0 when the pole $\alpha$ moves towards 0 .

Then, let us compute the transfer function $e(s) / \tau(s)$ in the presence of the additive uncertainty $\Delta_{a}(s)$. The obtained transfer function, called $\mathrm{H}_{2}$, is given by

$$
\begin{aligned}
H_{2}(s) & =S G_{d} G_{a} G_{p_{n}}\left(\frac{s^{2}}{(s-\alpha)^{2}}-1\right)-\Delta_{a} G_{a} G_{d} S \\
& =H_{1}-\Delta_{a} G_{a} G_{d} S .
\end{aligned}
$$

Finally, the effect of the uncertainty $\Delta_{a}$ on the closed-loop performance can be quantified computing the difference between $H_{2}$ and $H_{1}$, called $H_{\Delta}$, as

$$
H_{\Delta}=-\Delta_{a} G_{a} G_{d} S .
$$

Thus, (11) shows that the effects of the modeling error $\Delta_{a}$ are attenuated by the sensitivity function of the feedback loop. A 


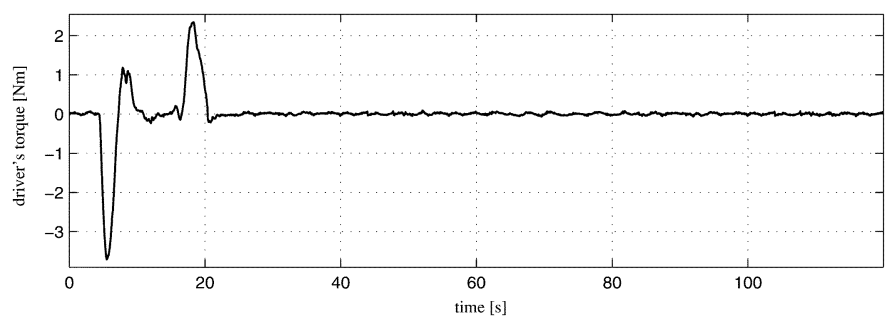

Fig. 6. Simulation results: driver's torque $\tau$ on the steer.

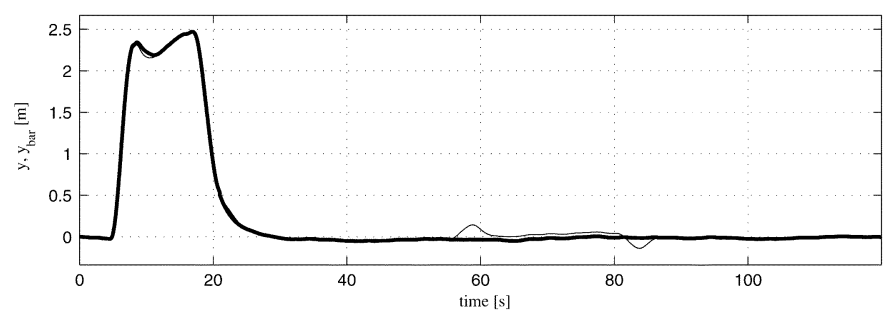

Fig. 7. Simulation results: $\bar{y}$ (thick line) and $y$ (thin line).

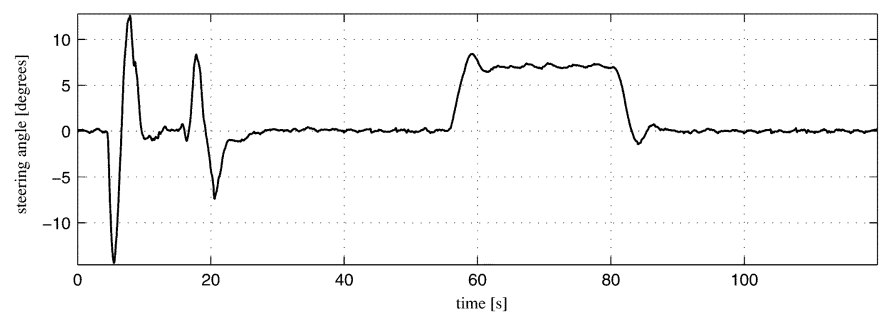

Fig. 8. Simulation results: steering angle.

quantitative evaluation of the robustness of the proposed 2-DOF structure is provided by the simulations presented in Section V.

\section{Simulation Results AND Discussion}

Here, we report the simulation results obtained with the proposed 2-DOF control structure of Fig. 4, where the mathematical model of the plant is the one presented in Section II and the lane-keeping controller designed in [21] is used for $C_{1}(s)$. The test track consists of a straight section followed by a curve with radius $R \approx 1000 \mathrm{~m}$. A double-lane-change maneuver has been performed when the car is driving along the straight section. The driver's torque in the simulation is an experimental torque sequence obtained from a double lane change performed by a test driver on an Italian highway driving the vehicle provided by FIAT S.p.A. The feedback output $y$ and the signal $\bar{y}$ are compared in Fig. 7 while Figs. 6 and 8 show the driver's torque $\tau$ and the steering angle $\delta_{v}$, respectively. Since the vision system is subject to a change in the measurement reference system during a lane change (see Section IV-D), the acquired signals $y$ and $\bar{y}$ should show a discontinuity. However, in order to ease the plots interpretation, data presented on Fig. 7 were postprocessed to eliminate the discontinuity of the measured $y$ and $\bar{y}$. From time $t=0 \mathrm{~s}$ to time $t \approx 55 \mathrm{~s}$, the vehicle is on a straight section. At time $t=4.5 \mathrm{~s}$, the driver applies a torque on the steer (see Fig. 6) to perform a double lane change which ends at time $t \approx 20 \mathrm{~s}$; as can be seen from Fig. 7, during this maneuver, the signal $\bar{y}$ is quite close to the output signal $y$ which means that the driver has full control of

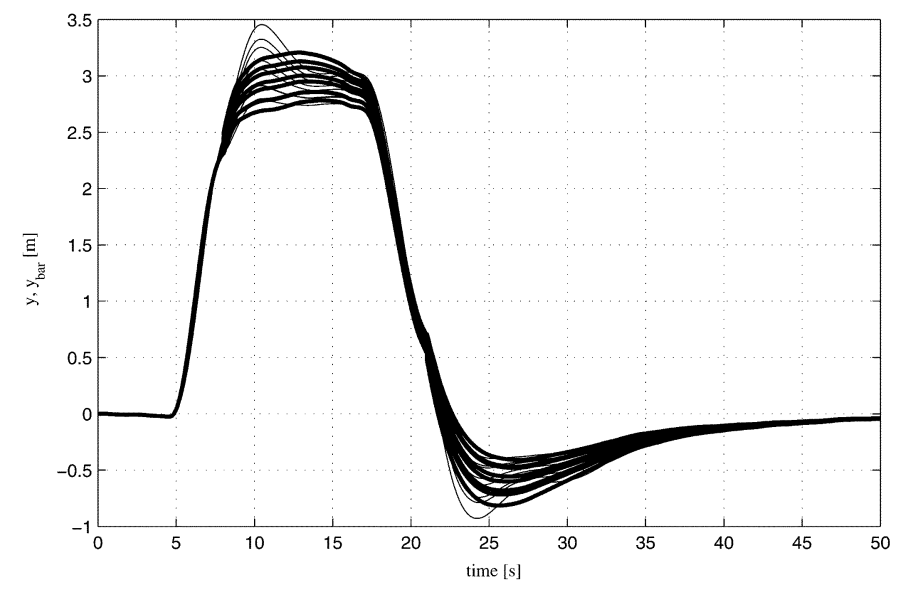

Fig. 9. Simulation results: $\bar{y}$ (thick line) and $y$ (thin line) for some values of $\gamma \in \Gamma$ and $v_{x}=60 \mathrm{~km} / \mathrm{h}$.

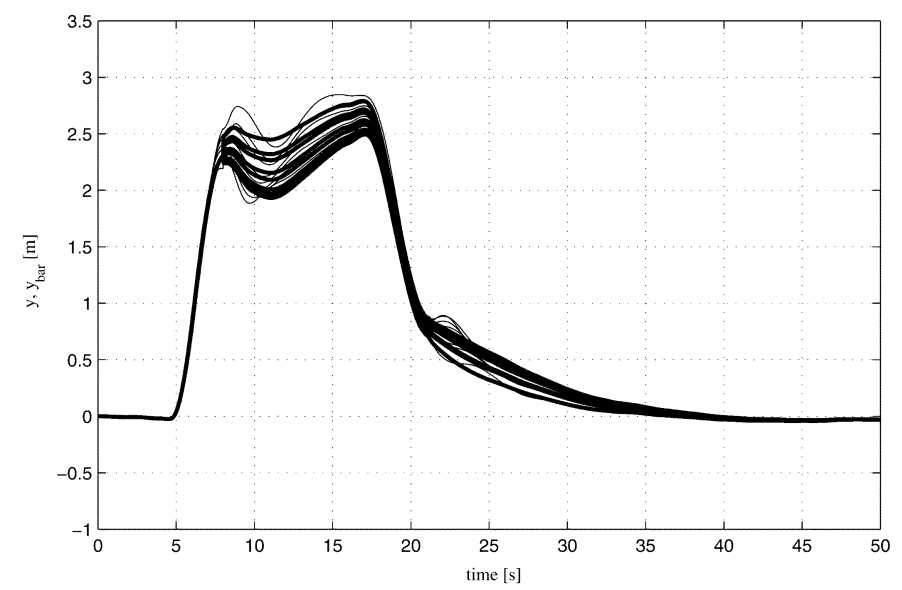

Fig. 10. Simulation results: $\bar{y}$ (thick line) and $y$ (thin line) for some values of $\gamma \in \Gamma$ and $v_{x}=120 \mathrm{~km} / \mathrm{h}$.

the vehicle during this stage [specification (S3)]. At the end of the double-lane-change maneuver, the driver's torque $\tau$ rapidly decreases to zero and the signal $\bar{y}$, i.e., the output of the designed filter $C_{2}$, goes to zero trough a smooth transient; during such a transient, the lane-keeping controller $C_{1}$ drives the vehicle to the centerline of the lane through the tracking of the signal $\bar{y}$ and, at the end of the transient, the lane-keeping task is resumed smoothly [specification (S4)]. From time $t \approx 55 \mathrm{~s}$ to time $t \approx 88 \mathrm{~s}$, the vehicle is driven by the lane-keeping system along a curve of radius $R \approx 1000 \mathrm{~m}$. During the curve, the driver does not operate on the steer, thus, as expected, the signal $\bar{y}$ approaches the value zero and the output $y$ of the system, which is the position error with respect to the centerline of the lane measured at the look-ahead distance $L$, is suitably regulated by the controller $C_{1}$ [specification (S1)]. The above simulations have been performed at the velocity $v_{x}=90 \mathrm{~km} / \mathrm{h}$. The effect of the parameters uncertainty on the performance of the proposed 2-DOF control system is analyzed in Figs. 9 and 10. Such figures shows the comparison between the feedback output $y$ and the signal $\bar{y}$ during a double lane change for a number of different values of the parameters $m_{v}, I_{\psi}, c_{r}$, and $c_{f}$. Fig. 9 presents the results obtained for a double lane change performed at the velocity $v_{x}=60 \mathrm{~km} / \mathrm{h}$, while Fig. 10 presents 


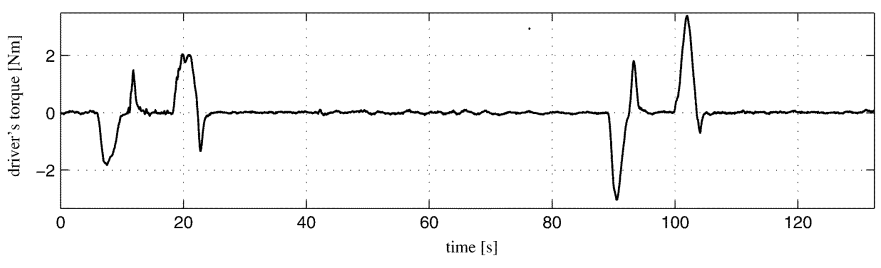

Fig. 11. Experimental results: driver's torque $\tau$ on the steer.

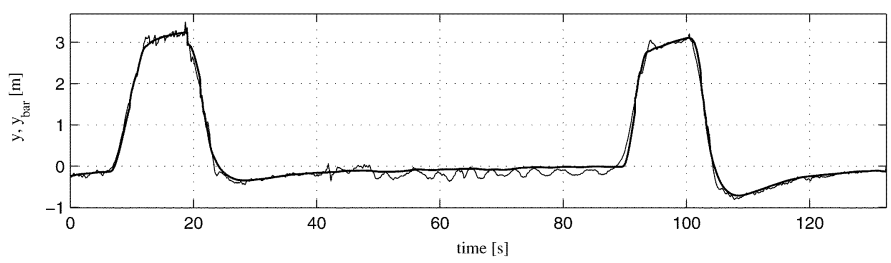

Fig. 12. Experimental results: $\bar{y}$ (thick line) and $y$ (thin line).

the results obtained for a double lane change performed at the velocity $v_{x}=120 \mathrm{~km} / \mathrm{h}$. It must be noted that in all of the simulations it was assumed that, coherently with the actual maneuver performed by the test driver on the real vehicle, the lane changes are always performed at time $t=8 \mathrm{~s}$ and $t=22 \mathrm{~s}$, respectively. In such a way, for each value of the uncertain parameters, the simulation study provides a different value to which the state variable $q$ has to be reset. Due to this fact, for each value of the uncertain parameters, we have a different signal $\bar{y}$ although the filter $C_{2}$ has been designed on the basis of the nominal plant $G_{p_{n}}$. As can be seen from such figures, the presence of uncertainty in the parameters causes only a slight degradation of the performance.

\section{EXPERIMENTAL RESULTS AND DISCUSSION}

Here, we report the experimental results obtained testing the controlled system along Italian highways. The proposed 2-DOF structure has been implemented on a FIAT Brava 1600 ELX provided by Centro Ricerche Fiat. The test track consists of two straight sections with a curve with radius $R \approx 800 \mathrm{~m}$ in between. Double-lane-change maneuvers have been performed when the car was driving along the straight sections. The feedback output $y$ and the signal $\bar{y}$ are compared in Fig. 12 while Figs. 11, 13, and 14 show the driver's torque $\tau$, the steering angle $\delta_{v}$, and the vehicle velocity $v_{x}$, respectively. Since the vision system is subject to a change in the measurement reference system during a lane change (see Section IV-D), the acquired signals $y$ and $\bar{y}$ should show a discontinuity. However, in order to ease the plots interpretation, data presented on Fig. 12 were postprocessed to eliminate the discontinuity of the measured $y$ and $\bar{y}$. From time $t=0 \mathrm{~s}$ to time $t \approx 48 \mathrm{~s}$, the vehicle is on a straight section. At time $t=6 \mathrm{~s}$, the driver applies a torque on the steer (see Fig. 11) to perform a double lane change which ends at time $t \approx 25 \mathrm{~s}$; as can be seen from Fig. 12, during this maneuver, the signal $\bar{y}$ is quite close to the output signal $y$ which means that the driver has full control of the vehicle during this stage [specification (S3)]. At the end of the double lane change maneuver the driver's torque $\tau$ rapidly decrease to zero and the signal $\bar{y}$, output of the designed filter $C_{2}$, goes to zero trough a smooth transient; during such a transient, the lane-keeping controller $C_{1}$ drives the vehicle to the centerline

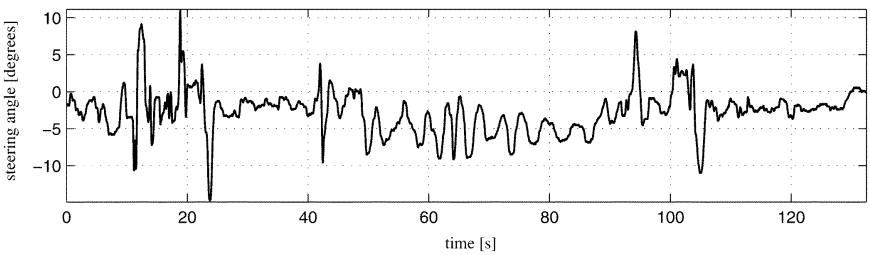

Fig. 13. Experimental results: steering angle.

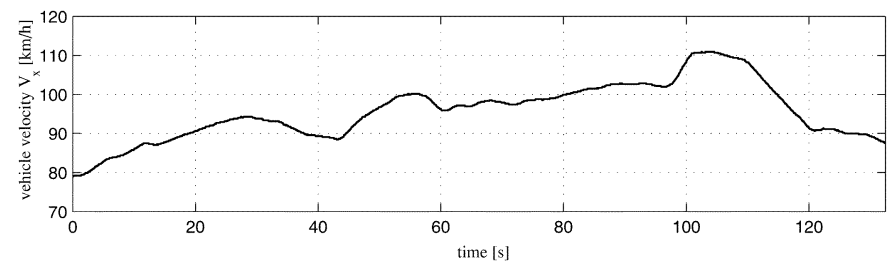

Fig. 14. Experimental results: vehicle velocity.

of the lane through the tracking of the signal $\bar{y}$ and, at the end of the transient, the lane-keeping task is resumed smoothly [specification (S4)]. From time $t \approx 50 \mathrm{~s}$ to time $t \approx 90 \mathrm{~s}$, the vehicle is driven by the lane keeping system along a curve of radius $R \approx 800 \mathrm{~m}$. During the curve, the driver does not operate on the steer, thus, as expected, the signal $\bar{y}$ approaches the value zero and the output $y$ of the system, which is the position error with respect to the centerline of the lane measured at the look-ahead distance $L$, is suitably regulated by the controller $C_{1}$ [specification (S1)]. Further, at time $t \approx 90 \mathrm{~s}$, a new double-lane-change maneuver is performed by the driver; as can be seen in Fig. 12, the lane-keeping task is smoothly released [specification (S2)], and the vehicle is controlled by the driver until time $t \approx 105 \mathrm{~s}$. Finally, the vehicle is driven again to the lane centerline.

\section{CONCLUSION}

In this paper, we addressed the problem of combining automatic lane-keeping and driver's steering for either obstacle avoidance or lane-change maneuvers for passing purposes or any other desired maneuvers, through a closed-loop control strategy. The automatic lane-keeping control loop is never opened, and no on/off switching strategy is used. During the driver's maneuver, the vehicle lateral dynamics is controlled by the driver himself through the vehicle steering system. When there is no driver's steering action, the vehicle center of gravity tracks the center of the traveling lane thanks to the automatic lane-keeping system. At the beginning (end) of the maneuver, the lane-keeping task is released (resumed) safely and smoothly. Simulation and experimental results along Italian highways paths obtained with the proposed 2-DOF structure on a FIAT Brava 1600 ELX provided by Centro Ricerche Fiat, showed the fulfillment of given specifications.

\section{ACKNOWLEDGMENT}

The authors would like to thank S. Campo and A. Chinu of Centro Ricerche Fiat for the fruitful discussions during the development of the research.

\section{REFERENCES}

[1] J. Tanaka, S. Ishida, H. Kawagoe, and S. Kondo, "Workload of using a driver assistance system," in Proc. IEEE Intell. Transportation Syst. Conf., 2000, pp. 382-386. 
[2] R. Fenton and R. Mayhan, "Automated highway studies at the ohio state university-An overview," IEEE Trans. Veh. Technol., vol. 40, no. 1, pp. 100-113, Feb. 1991.

[3] S. Shladover, C. Desoer, K. Hedrik, M. Tomizuka, J. Walrand, W. Zhang, D. McMahon, H. Peng, S. Sheikholeslam, and N. McKeown, "Automated vehicle control developements in the path program," IEEE Trans. Veh. Technol., vol. 40, no. 1, pp. 114-130, Feb. 1991.

[4] S. Patwardhan, H. Tan, and J. Guldner, "A general framework for automatic steering control: System analysis," in Proc. Amer. Control Conf., 1997, pp. 1598-1602.

[5] J. Kosěcá, R. Blasi, J. Taylor, and J. Malik, "A comparatative study of vision-based lateral control strategies for autonomos highway driving," in Proc. IEEE Int. Conf. Robot. Autom., 1998, pp. 1903-1908.

[6] P. Hingwe and M. Tomizuka, "A variable look-ahead controller for lateral guidance of four wheeled vehicles," in Proc. IEEE Amer. Control Conf., 1998, pp. 31-35.

[7] J. Wang and M. Tomizuka, "Robust $h_{\infty}$ lateral control of heavy-duty vehicles in automated higway system," in Proc. Amer. Control Conf., 1999, pp. 3671-3675.

[8] M. Tai, P. Hingwe, and M. Tomizuka, "Modeling and control of steering system of heavy vehicles for automated highway systems," IEEE/ASME Trans. Mechatron., vol. 9, no. 4, pp. 609-618, Aug. 2004.

[9] M. Tomizuka, M. Tai, J.-Y. Wang, and P. Hingwe, "Automated lane guidance of commercial vehicles," in Proc. IEEE Int. Conf. Control Appl., 1999, pp. 1359-1364.

[10] S. Ibaraki, S. Suryanarayanan, and M. Tomizuka, "Design of Luemberger state observer usign fixed hoc optimization and its application to fault detection in lane-keeping control of automated vehicles," IEEE/ ASME Trans. Mechatron., vol. 10, no. 1, pp. 34-42, Feb. 2005.

[11] S. Suryanarayanan, M. Tomizuka, and T. Suzuki, "Design of simultaneously stabilizing controllers and its application to faul-tolerant lanekeeping controller design for automated vehicles," IEEE Trans. Control Syst. Technol., vol. 12, no. 3, pp. 329-339, May 2004.

[12] J. Ackermann, J. Guldner, W. Sienel, R. Steinhauser, and V. Utkin, "Linear and nonlinear controller design for robust automated steering," IEEE Trans. Control Syst. Technol., vol. 3, no. 1, pp. 132-143, Jan. 1995.

[13] J. Guldner, W. Sienel, J. Ackermann, S. Patwardhan, and T. Bünte, "Robust automatic steering control for look-down reference systems with front and rear sensors," IEEE Trans. Control Syst. Technol., vol. 7, no. 1, pp. 2-11, Jan. 1999.

[14] R. Byrne, C. Abdallah, and P. Dorato, "Experimental results in robust lateral control of highway vehicles," IEEE Control Syst. Mag., pp. 70-76, 1998.

[15] Q. Wang, M. Oya, and T. Kobayashi, "Adaptive steering control scheme for combination vehicles to track target lane," in Proc. SICE-ICASE Int. Joint Conf., 2006, pp. 1790-1795.

[16] P. Falcone, F. Borrelli, J. Asgari, H. Tseng, and D. Hovrat, "Predictive active steering control for autonomous vehicle systems," IEEE Transaction on Control Syst. Technol., vol. 15, no. 3, pp. 566-580, May 2007.

[17] C. Hatipoğlu, K. Redmill, and Ü. Özgüner, "Steering and lane change: A working system," in Proc. IEEE Intell. Transportation Syst. Conf., 1997, pp. 272-277.

[18] A. Broggi, M. Bertolozzi, A. Fascioli, C. G. L. Bianco, and A. Piazzi, "The argo autonomous vehicle's vision and control systems," Int. J. Intell. Control Syst., vol. 3, no. 4, pp. 409-441, 1999.

[19] V. Cerone, A. Chinu, and D. Regruto, "Experimental results in vision-based lane keeping for highway vehicles," in Proc. Amer. Control Conf., 2002, pp. 3126-3131.

[20] V. Cerone and D. Regruto, "Vehicle lateral controller design exploiting properties of sito systems," in Proc. Amer. Control Conf., 2003, pp. $4365-4370$.
[21] V. Cerone and D. Regruto, "Robust performance controller design for vehicle lane keeping," presented at the European Control Conf. (ECC'03), Cambridge, U.K., Sep. 2003.

[22] S. Tsugawa, "Vision-based vehicles in japan: Machine vision systems and driving control systems," IEEE Trans. Ind. Electron., vol. 41, no. 4, pp. 398-405, Aug. 1994.

[23] C. Hatipoğlu, Ü. Özgüner, and K. Redmill, "Automated lane change controller design," IEEE Trans. Intell. Transportation Syst., vol. 4, no. 1, pp. 13-22, Jan. 2003.

[24] C. Hatipoğlu, Ü. Ozgüner, and K. A. Ünyelioğlu, "On optimal design of a lane change controller," in Proc. IEEE Intell. Vehicles Symp., 1995, pp. 436-441.

[25] R. Fenton and I. Selim, "On the optimal design of an automotive lateral controller," IEEE Trans. Veh. Technol., vol. 37, no. 2, pp. 108-113, May 1988.

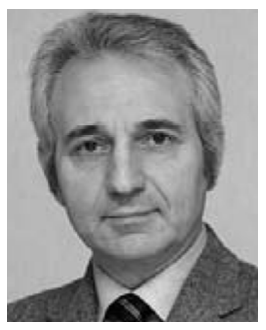

Vito Cerone (M'07) received the Laurea degree in electronic engineering and the Ph.D. degree in system engineering from the Politecnico di Torino, Torino, Italy, in 1984 and 1989, respectively.

$\mathrm{He}$ is an Associate Professor with the Politecnico di Torino, where he teaches analysis of dynamical systems, feedback control system design, and analysis and design of physiological systems. His main research interests are in system identification, parameter estimation, control, optimization, and their applications.

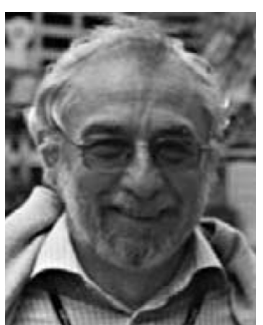

Mario Milanese (SM'04) received the degree in electronic engineering from Politecnico di Torino, Torino, Italy, in 1967.

From 1968 to 1972, he was Teaching Assistant with Politecnico di Torino, and, from 1972 to 1980, he was an Associate Professor of System Theory with Università di Torino. Since 1980, he has been a Full Professor of System Theory with Politecnico di Torino. From 1982 to 1987, he was head of the Dipartimento di Automatica e Informatica at Politecnico di Torino. His research interests include robust identification, prediction and control of uncertain systems, and applications to biomedical, automotive, aerospace, financial, and environmental problems. $\mathrm{He}$ is the author of more than 180 papers in international journals and conference proceedings. He is editor of the books Robustness in Identification and Control (Plenum, 1989) and Bounding Approaches to System Identification (Plenum, 1996).

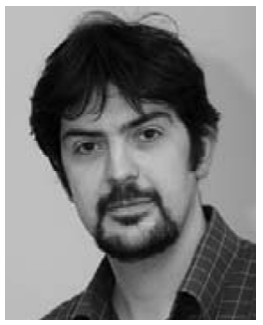

Diego Regruto (M'04) received the Laurea degree in electronic engineering and the Ph.D. degree in system engineering from Politecnico di Torino, Torino, Italy, in 2000 and 2004, respectively.

$\mathrm{He}$ is currently an Assistant Professor with the Dipartimento di Automatica e Informatica, Politecnico di Torino. His main research interests are in the fields of system identification and robust control, with application to automotive problems. 